\title{
AUMENTO DE PRODUTIVIDADE DAS LINHAS DE ACABAMENTO (OTIMIZAÇÃO DO PÁTIO DE BOBINAS BQ-1) DA ARCELORMITTAL TUBARÃO*
}

Luis Roberto Zorzanelli ${ }^{1}$

Flávio de Araujo Porto

\section{Resumo}

Esse trabalho tem como objetivo apresentar o projeto de melhoria da automação das pontes rolantes (PRs) e arranjo do pátio de bobinas BQ1, onde estão localizadas as linhas de acabamento (LAs)(Encruamento e Corte) como parte do projeto maior de aumento de produtividade destas plantas. Percebeu-se que a atuação na logística interna do pátio e na interface com as PRs poderia obter bons resultados nas movimentações de bobinas. O pátio tem áreas de recebimento e despacho de bobinas e quatro PRs e, portanto, grande número de movimentos possíveis, o grande desafio foi capturar estas informações e desenhar uma solução que atendesse as expectativas. Desta forma se buscou realizar um levantamento de dados minucioso para abrangendo o sistema existente, as características físicas e o modo operacional para definição de regras de movimentação otimizadas. As ações implementadas foram a revisão completa do arranjo do pátio e redefinição de regras de movimentação, e a implantação de terminal embarcado nas PRs para instruir as movimentações além de fornecer informações e dar autonomia para a tomada de algumas decisões controladas.

Palavras-chave: Pátio de bobinas; Linhas de acabamento (LAs); Arranjo do pátio; Terminal embarcado.

\section{IMPROVEMENT OF PRODUCTIVITY OF FINISHING LINES (COIL YARD OPTIMIZATION) OF ARCELORMITTAL TUBARAO}

\section{Abstract}

This paper aims to present the automation improvement project of cranes (PRs) and BQ1 coil yard arrangement, where are located the finishing lines (LAs) as part of the larger project to increase productivity of these plants. It was felt that the work on the internal logistics of the courtyard and the interface with the PRs could get good results in coil movement. The courtyard has receiving and dispatching coils and four PRs, and therefore, a large number of possible movements, the big challenge was to capture this information and design a solution that meets the expectations. In this way we sought to survey of detailed data to covering the existing system, the physical characteristics and the operating mode for setting optimized handling rules. The actions taken were full of the courtyard arrangement review and redefinition of movement rules, and terminal deployment in embedded PRs to instruct the movement and provide information and empower for making certain subsidiaries decisions.

Keywords: Coil Yard; Hot Skin Pass Line and Divide Line; Yard arrangement; Crane embedded computer.

1 Engenheiro Especialista de Automação, Gerência de Área de Engenharia de Automação, ArcelorMittal Tubarão, Serra, ES, Brasil.

2 Engenheiro de Operação, Gerência de Área de Acabamento de Bobinas, ArcelorMittal Tubarão, Serra, ES, Brasil 


\section{INTRODUÇÃO}

As linhas de acabamento de bobinas da ArcelorMittal Tubarão (LAs), formada por uma linha de encruamento e uma linha de tesoura, são responsáveis pelo processamento de bobinas produzidas no laminador de tiras a quente (LTQ) com a finalidade de melhoria da qualidade superficial e/ou divisão. Estas linhas com capacidade nominal de $750 \mathrm{kt} / \mathrm{ano}$ vinham perdendo produtividade a partir de 2009 , com o aumento de produção do LTQ, devido a fatores como o aumento de participação de materiais críticos e para tratamento de defeitos na LA e LT, a implantação de uma linha de corte e amostra (LCA) tirando material de alta produtividade da LT e outros motivos diversos.

Desta forma no final de 2014 se iniciou um projeto para recuperação de produção tomando como referência o ano de maior produção 2007 (1670kt/ano) contra uma produção estimada de 1172Kt/ano em 2014. A meta estabelecida foi de uma recuperação de 200kton em um ano. Foi formado um grupo multidisciplinar com as diversas especialidades envolvidas na busca da meta estabelecida.

Coube a área de automação de processos a análise da logística interna do pátio BQ1, onde se encontram instaladas as LAs, que identificou oportunidade na melhoria da automação das pontes rolantes e no arranjo do pátio de modo a ganhar agilidade no recebimento, movimentação de carga e descarga das LAs e despacho de bobinas, reduzir o retrabalho com relocações, reduzir falhas de tracking e reduzir tempo de permanência de carretas no pátio.

O pátio de bobinas BQ1 é gerenciado por um sistema de Nível 2 que mantinha suas características praticamente originais desde sua implantação com o startup das LAs em 2002. Mas as condições operacionais tinham sofrido diversas alterações desde então. Por isso foi necessário um estudo minucioso e detalhado de todo o modo operacional vigente para a elaboração da especificação detalhada das novas funcionalidades.

\section{MATERIAIS E MÉTODOS}

Para atendimento aos objetivos definidos na análise inicial, o projeto foi dividido em 2 grandes etapas.

$>$ Levantamento de dados

- Análise das funcionalidades do sistema existente

- Análise do layout do pátio e equipamentos móveis

- Estudo detalhado do modo operacional vigente

- Análise de requisitos e descrição funcional das melhorias

$>$ Desenvolvimento da aplicação

- Projeto de software

- Desenvolvimento das funcionalidades

- Testes integrados

- Treinamento

- Implantação

\subsection{Levantamento de Dados}

- Análise das funcionalidades do sistema existente

O sistema de gerenciamento de Nível 2 (HDCYM) é responsável pelo gerenciamento dos pátios de processo de bobinas, linhas de acabamento e pátios de despacho de 
bobinas, tendo como principais funcionalidades o recebimento, processamento nas LAs, armazenamento, rastreamento, interface com equipamentos de Nível 1 (pontes rolantes, carro de transferência de bobinas e linhas de acabamento) e despacho das bobinas através de caminhões. Este sistema é responsável pelos pátios $B Q 1, B Q 2$, BQ4, BQ5, BQ6, BQ8 e pelas linhas de encruamento (LA) e linha de corte (LT).

Na figura 1 pode ser verificado o layout geral do pátio.

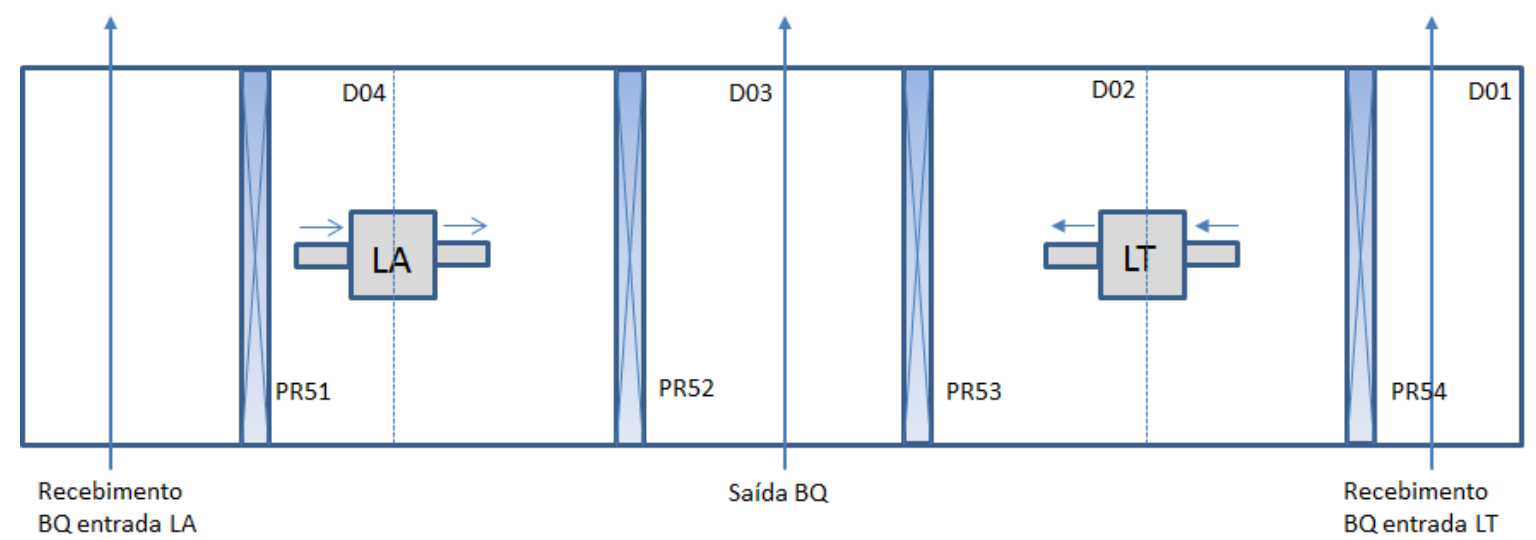

Figura 1. - Layout geral Pátio BQ1

O sistema de Nível 2 era responsável pelo posicionamento das bobinas no pátio e pela definição e envio de instruções de movimentação de bobinas para as pontes rolantes (PRs), que garantiam a posição correta das bobinas desde que todas as operações fossem executadas pelas PRs conforme instrução enviada, mas devido ao grande volume de bobinas e a dinâmica do processo operacional os critérios atuais de armazenamento de BQs não estavam atendendo plenamente a operação sendo necessárias movimentações em manual, que causavam constantes falhas de tracking e retrabalho operacional. As regras e critérios de definição de setup de movimentação das BQs não atendiam mais as necessidades operacionais. Desta forma, os operadores das PRs após movimentarem as BQs solicitavam via rádio ao controlador do pátio a correção manual da posição.

\section{$\bullet$}

- Análise do layout do pátio e equipamentos móveis

No sistema de Nível 2 o pátio BQ1 é dividido em quatro áreas de armazenamento (D01, D02, D03 e D04), 1 área de entrada da LT EWB (D05), 1 área de saída da LT DWB (D05), 1 área de entrada da LA EWB (D06), 1 área de saída da LA DWB (D06), 1 área de recebimento/despacho rodoviário (D08), 1 área de virador de bobinas (D09), 1 área de embalagem de bobinas (D10), 1 área de cintamento axial de bobinas (D11) e 1 área de transferência de bobinas para o BQ2 (D12). A área de recebimento/despacho rodoviário (D08) é composta de 1 posição de entrada na LT, 1 posição de entrada na LA e 3 posições de saída de bobinas no meio do pátio. As bobinas são armazenadas em duas camadas. O pátio dispõe de 4 pontes rolantes com capacidade de $40 \mathrm{t}$, sendo duas atendendo as entradas e duas atendendo as saídas.

$\mathrm{Na}$ figura 2 é mostrado o mapeamento do pátio BQ1 visualizado pelas telas do sistema de Nível 2. 

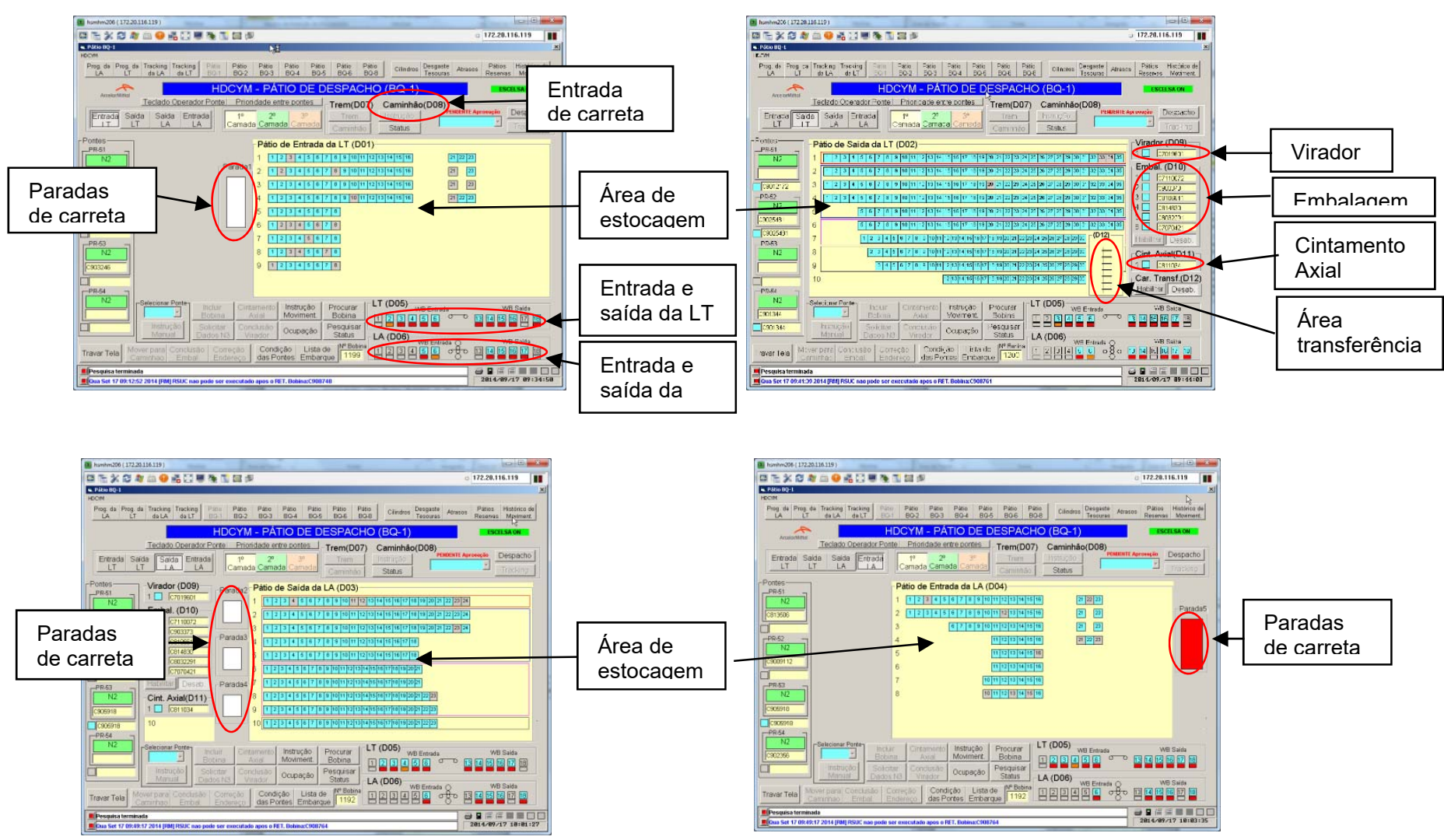

Figura 2. -Pátio BQ1 - Sistema de Nível 2

Foi realizada visitas ao pátio para confirmação de número de berços e alas, medições de distância entre berços e entre alas, e dimensionamento da abertura da tenaz utilizada, com objetivo de recalcular as restrições físicas de armazenamento de bobinas em função da dimensão, cujas informações serão utilizadas para o cálculo das restrições lógicas de distribuição das bobinas no pátio.

Na figura 3 é mostrado o layout físico do pátio BQ1.

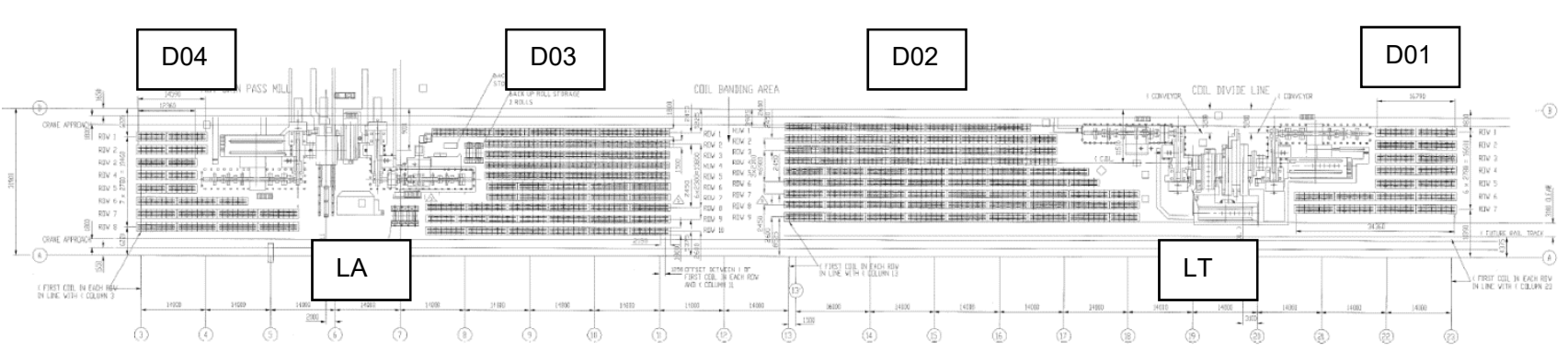

Figura 3. - Layout físico Pátio $B Q 1$

Na figura 4 são apresentados os cálculos para as áreas de entrada do pátio. 
Entrada da LA e LT

Distância entre berços $1730 \mathrm{~mm}$

Considerações:

- Largura da garra da tenaz $=500 \mathrm{~mm}$

- Distância segurança-150mm

- Abertura máxima da tenaz - Máx 3170mm, Min 1200mm

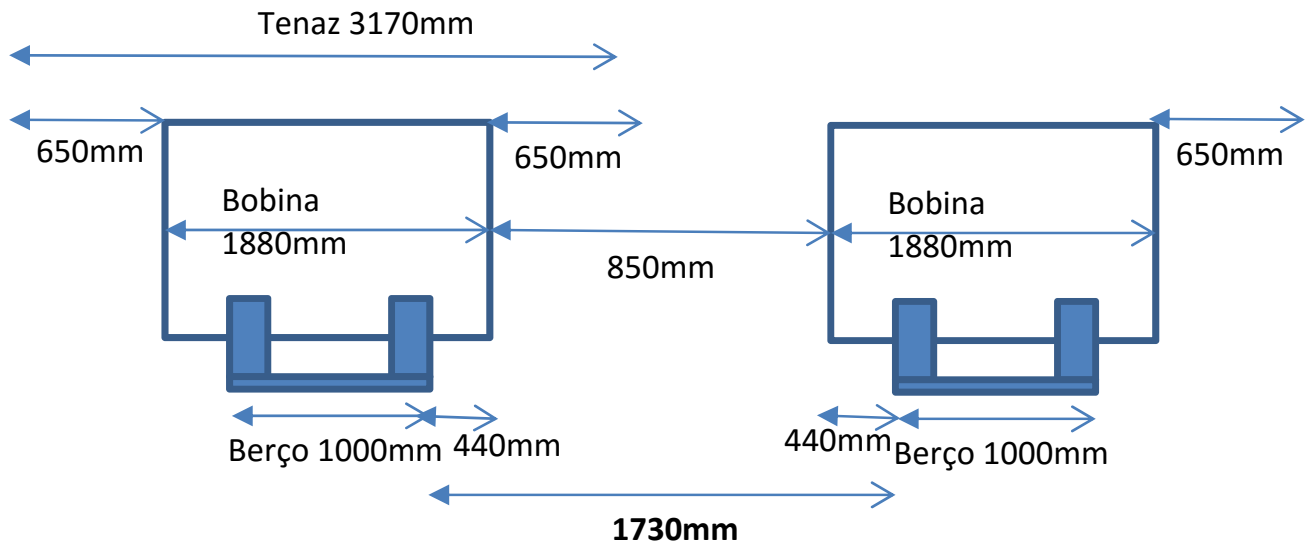

Figura 4. - Cálculos de dimensão máxima de BQ

- Estudo detalhado do modo operacional vigente

Foram realizadas diversas reuniões com especialistas e operadores da área, além de acompanhamento dos procedimentos operacionais desde o recebimento da bobina até o despacho da mesma, visando um entendimento das condições operacionais.

Basicamente foram observados os seguintes pontos:

- Os operadores das PRs precisavam tomar muitas decisões de movimentação, mas não tinham informações suficientes para esta ação. Para contornar esta situação eles levavam para a PR uma lista de BQs que deveriam movimentar e utilizavam o rádio para se comunicar com o operador controlador do pátio com muita frequência. Este fato ocorria principalmente na função de carregamento de carretas para despacho;

- O operador controlador do pátio passava uma boa parte do tempo atualizando manualmente a posição das BQs no sistema;

- O operador controlador do pátio precisava buscar informações frequentes no sistema corporativo para atualizar as informações de BQs para despacho;

- Como as operações de atualização de endereço de BQs no sistema eram normalmente realizadas algum tempo após a movimentação real da $B Q$, falhas de tracking eram constantes;

Foi realizado também um estudo estatístico do mix de produção dos últimos 15 meses visando avaliar as dimensões de BQs produzidas.

- Análise de requisitos e descrição funcional das melhorias

O resultado das etapas anteriores foi um conjunto de informações que embasaram as propostas de melhorias, descritas abaixo. 
- Atualização periódica do sistema de Nível 2 de informações relevantes para a tomada de decisão de recebimento, movimentação e despacho de bobinas (Prioridade, Status, Modal de despacho, data de prontidão, etc.);

- Revisão de todas as regras e critérios de decisão de armazenamento buscando melhor ocupação do pátio e agrupamento de BQs, sempre com foco em facilidade de movimentação com menor relocação, respeitando-se restrições físicas e de segurança. A figura 5 apresenta as dimensões máximas de BQs por ala na área D02.

D02
\begin{tabular}{|c|c|c|}
\hline Ala & $\begin{array}{c}\text { Largura } \\
\text { máxima }(\mathrm{mm})\end{array}$ & $\begin{array}{c}\text { Diâmetro externo } \\
\text { máximo }(\mathrm{mm})\end{array}$ \\
\hline 1,2 & 1880 & 2150 \\
\hline 3,4 & 1820 & 2150 \\
\hline 5,6 & 1670 & 2150 \\
\hline 7 & 1670 & 1890 \\
\hline 8,9 & 1820 & 1890 \\
\hline 10 & 1880 & 1890 \\
\hline
\end{tabular}

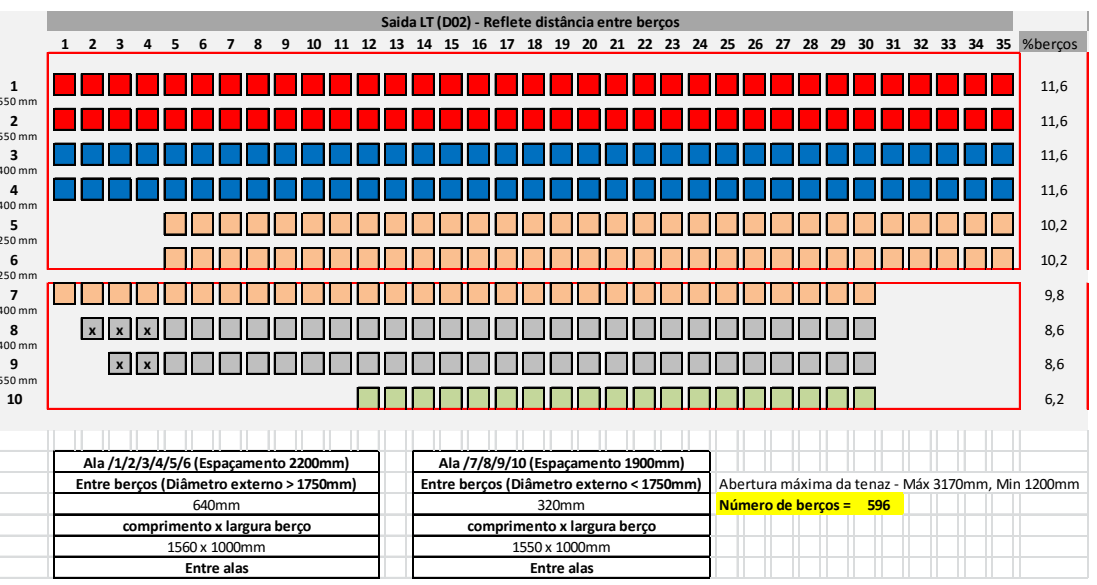

Figura 5. - Dimensões físicas área D02

Para maximizar a capacidade de alocação e flexibilidade de movimentação foi criado o critério lógico de armazenamento que é configurável, respeitando-se os critérios físicos e o mix de produção avaliado. A figura 6 representa esta configuração para a área D02.

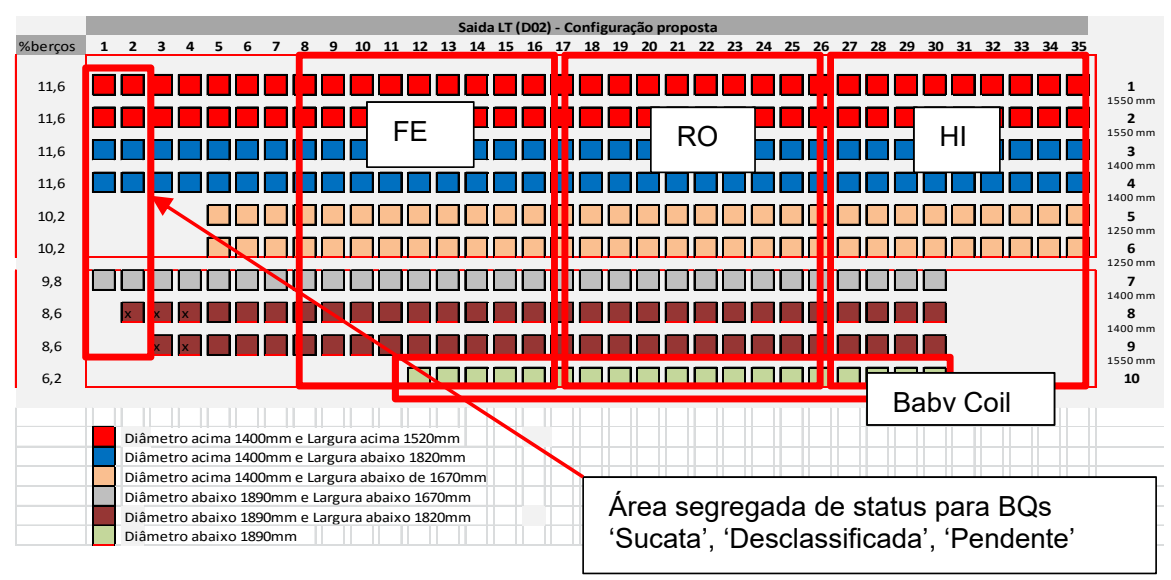

Figura 6. - Dimensões lógicas área D02

- Revisão de todas as regras e critérios de decisão de movimentação buscando enviar aos operadores das PRs instruções de movimentação otimizadas;

- Avaliação de soluções de sensoriamento para localização e detecção de posição nos três eixos cartesianos (X, Y e Z). Foram identificados equipamentos baseados em rádio frequência de longo e curto alcance, tais como radar, laser, RFID, além de encoders. As soluções analisadas, em geral, incluíam uma combinação de sensores, que dependiam do nível de automação desejado e das características do pátio (Layout irregular, armazenamento em duas camadas, 4 pontes rolantes em mesmo 
vão), que elevaram os custos significativamente. Desta forma optou-se num primeiro momento por utilizar os recursos existentes de detector de peso e sensor de bobina na tenaz, juntamente com instruções de movimentação mais assertivas que atenderiam os propósitos desejados. E depois num segundo momento aprofundar os estudos de automação, com objetivos não somente de rastreabilidade, mas também de operação automática ou remota das PRs.

- Implantação de terminais de operação nas PRs proporcionando ao operador de informações necessárias a qualquer tipo de movimentação sem a necessidade contato com o operador controlador. O sistema aplicativo embarcado em computadores industriais foi desenvolvido em C\# que se comunica como servidor de Nível 2 através de protocolo TCPIP em uma rede wireless existente no pátio.

A figura 7 apresenta as principais funcionalidades deste aplicativo.
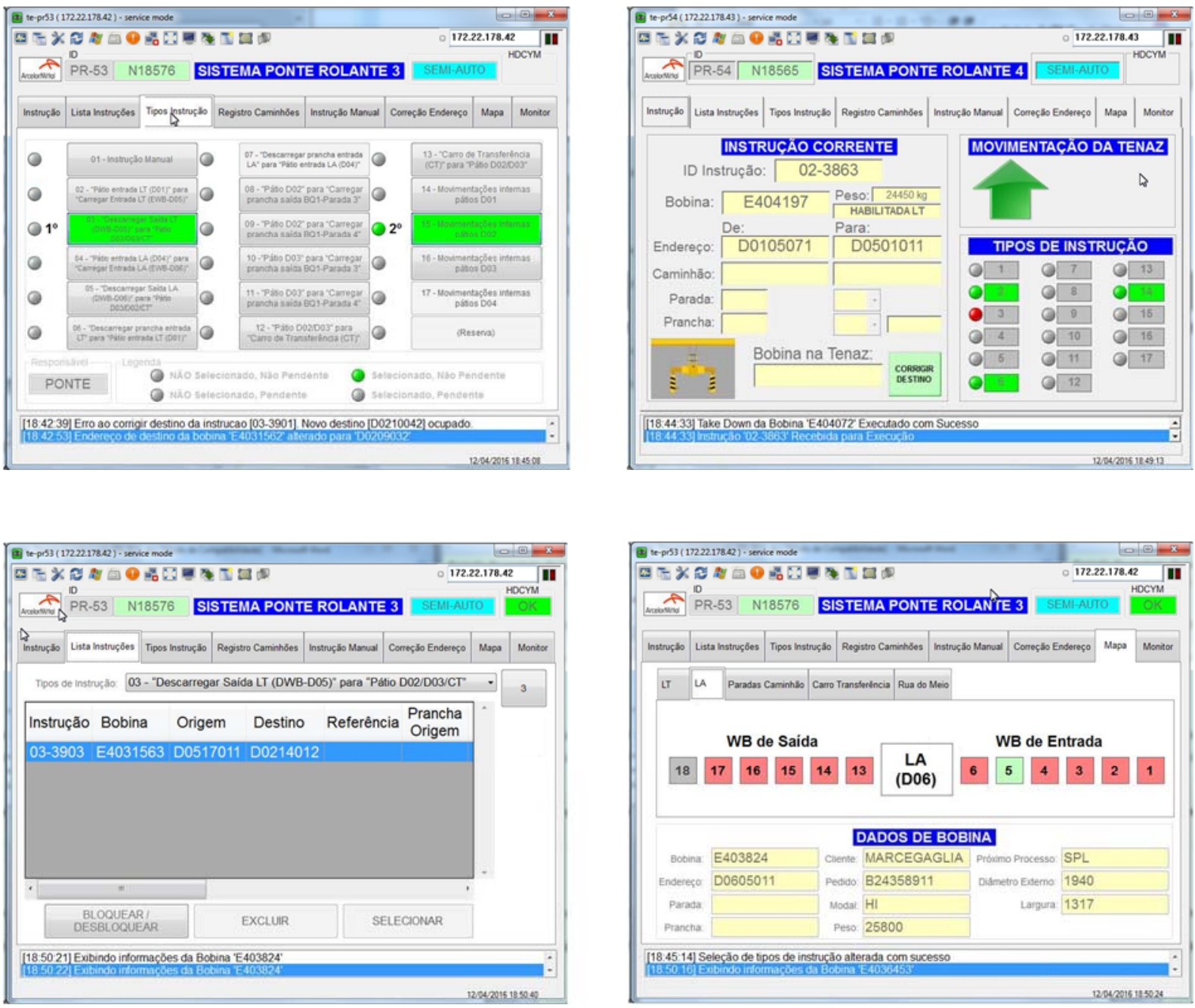

Figura 7. - Principais funcionalidades do sistema embarcado nas PRs

A figura 8 mostra um terminal instalado em uma PR. 


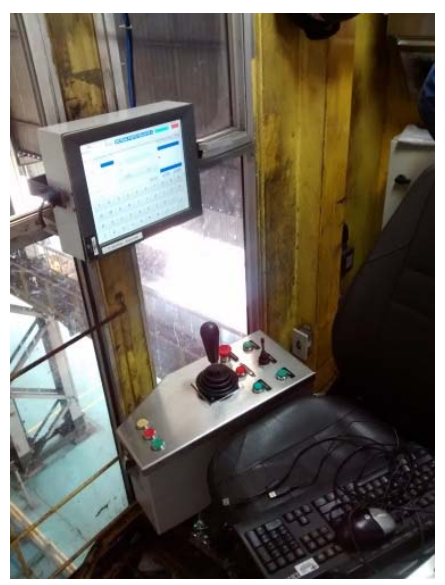

Figura 8. - Vista de um terminal na PR

\subsection{Desenvolvimento da Aplicação}

O desenvolvimento do sistema aplicativo das PRs e as adequações do aplicativo de Nível 2 foi realizado por uma empresa parceira, tendo como base as especificações geradas na etapa anterior. Esta etapa foi dividida em 4 blocos, a saber:

- Projeto de software -> Com base nas especificações geradas foi desenvolvido o detalhamento das funcionalidades que serviram de guia para o bloco seguinte;

- Desenvolvimento das funcionalidades -> O aplicativo das PRs foi desenvolvido em MS Visual Studio 2013 (C\#) para o SO Windows 7. O aplicativo do Nível 2 foi desenvolvido através da adequação do sistema existente em $C$ standard para o SO Tru64Unix e em MS Visual Basic 6.0 para o SO Windows XP;

- Testes integrados -> Ao final do desenvolvimento foram realizados os testes integrando todos os módulos e simulando todas as possíveis situações reais levantadas;

- Treinamento -> Etapa importante do projeto devido principalmente a utilização de tecnologia nova e mudança de hábito operacional, onde a responsabilidade do operador da PR aumenta;

- Implantação -> Para reduzir o risco de impacto operacional e de perda de produção nas LAs, o sistema foi implantado em duas fases, sendo a $1^{\mathrm{a}}$ nas PRs de entrada do pátio e LAs e a $2^{a}$ nas PRs de saída do pátio e LAs. Além disso, foi criado a possibilidade de "Ligar/Desligar" o sistema novo na $1^{\text {a }}$ fase. Esta estratégia se mostrou bastante acertada, pois proporcionou a possibilidade de consolidar o treinamento operacional, corrigir desvios na aplicação e incluir pequenas melhorias não observadas na etapa de especificação;

\section{RESULTADOS E DISCUSSÃO}

A $1^{\text {a }}$ fase do sistema foi implantada em setembro e a $2^{a}$ fase em outubro de 2015 . $O$ tempo de adaptação dos operadores durou aproximadamente 30 dias, sendo depois utilizado em período de 24/7.

O projeto global de recuperação de produção das LAs atingiu a meta no final de 2015. Estima-se que a contribuição da iniciativa deste trabalho contribuiu com $5 \%$ deste resultado. 


\section{Produtividade LA e LT}

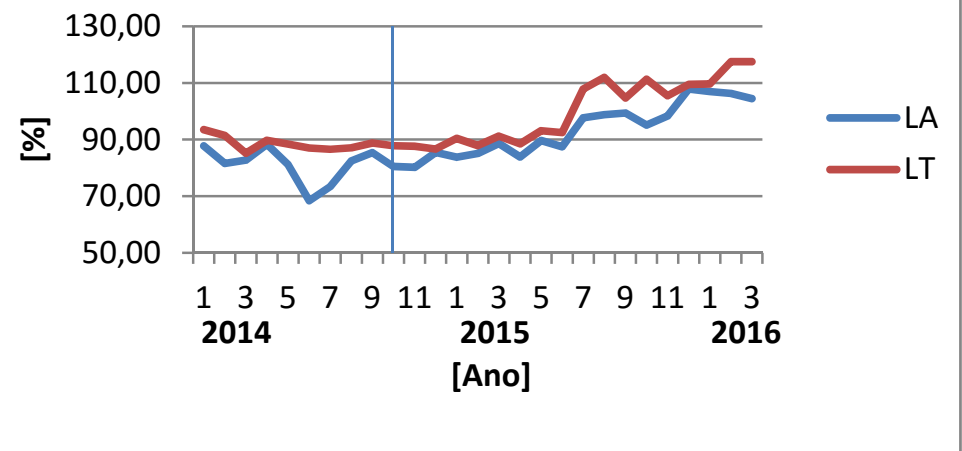

Figura 9. - Evolução da recuperação de produção das LAs

Dentre os principais benefícios deste projeto podemos citar:

- Melhoria nas informações disponíveis para os operadores das PRs, proporcionando mais autonomia na tomada de decisão

- Instruções de movimentação mais assertivas, reduzindo a necessidade de correções manuais, antes realizadas pelo operador controlador do pátio

- Melhoria na organização do pátio com melhor agrupamento de bobinas e redução de relocações

- Redução de falhas de tracking de bobinas observados na busca de bobinas para despacho

- Atualização tecnológica

\section{CONCLUSÃO}

O desenvolvimento e implantação deste projeto proporcionaram além dos benefícios já citados, a percepção pela área operacional de outras possibilidades de ganhos seja por aumento de produtividade ou racionalização de $\mathrm{HH}$. Devido a isso já foram solicitados estudos de viabilidade de implantação de sistema similar em outros pátios de bobinas da companhia.

\section{Agradecimentos}

À empresa Etaure TI \& Automação pela parceria e comprometimento no desenvolvimento e implantação deste projeto.

À equipe de infraestrutura de rede de dados da companhia pela presteza no atendimento e solução de questões relacionadas a comunicação de dados sem fio. 